\title{
Does Pen Help? A Real-World Outcomes Study of Switching from Vial to Disposable Pen Among Insulin Glargine-Treated Patients with Type 2 Diabetes Mellitus
}

\author{
Lin Xie, MS, MA, Steve Zhou, $\mathrm{PhD}^{2}$ Wenhui Wei, $\mathrm{PhD}^{2}$ Jasvinder Gill, $\mathrm{PhD}^{2}$ \\ Chunshen Pan, $\mathrm{PhD}, \mathrm{MSc}^{3}$ and Onur Baser, $\mathrm{PhD}^{1,4}$
}

\begin{abstract}
Objective: The study was designed to evaluate real-world data on clinical and economic outcome differences between patients with type 2 diabetes mellitus (T2DM) who use insulin glargine with vial-and-syringe delivery and those who switch to pen administration.

Subjects and Methods: This retrospective study analyzed medical and pharmacy claims information from the national managed-care IMPACT ${ }^{\circledR}$ database (Ingenix Inc., Salt Lake City, UT). Adults with T2DM treated with insulin glargine were evaluated. Clinical and economic outcomes over 1 year were compared between individuals who had converted from administering glargine via vial-and-syringe to the SoloSTAR ${ }^{\circledR}$ (sanofi-aventis U.S., Bridgewater, NJ) pen (Switchers) and patients who continued to use vial-and-syringe administration (Continuers). Patients from each cohort were matched using propensity score matching for a comparison sample.

Results: In total, 3,893 eligible patients were identified (665 Switchers and 3,228 Continuers), with a matched cohort with 603 patients in each group. Baseline characteristics were similar between groups. One-year treatment persistence was significantly higher with Switchers versus Continuers $(65.3 \%$ vs. $49.8 \% ; P<0.0001)$. Medication possession ratio was also significantly higher among Switchers (0.79 vs. $0.76 ; P=0.0173)$. Insulin use and glycemic control were similar between groups. Healthcare utilization and total costs were also similar between groups. Higher prescription costs among Switchers were offset by lower overall and diabetes-related outpatient and inpatient costs.

Conclusions: Switching from insulin glargine vial-and-syringe administration to pen delivery resulted in improved treatment adherence and persistence, with comparable clinical and economic outcomes.
\end{abstract}

\section{Introduction}

B ASAL INSULIN USE IS RECOMMENDED in patients with type 2 diabetes mellitus (T2DM) who are unable to achieve adequate glycemic control despite the use of oral antidiabetes drugs. ${ }^{1-3}$ Insulin has traditionally been administered using a syringe to extract insulin stored in a vial, ${ }^{1}$ but the anxiety, fear of pain, and social embarrassment associated with syringe-and-vial insulin may reduce insulin acceptance and treatment adherence. ${ }^{4-7}$ Alternatively, insulin may be delivered using prefilled disposable pen devices. Both patients and physicians prefer insulin pens because of enhanced discretion, ease of use, and convenience of self-injection. ${ }^{1,4,8-10}$ Pen devices also improve patient confidence in drug administration and glycemic control, which may also facilitate better adherence to insulin treatment. ${ }^{4}$
Several studies have investigated the delivery of insulin via disposable pen devices compared with vial-and-syringe delivery. ${ }^{5,11-15}$ However, not many have studied the effect of switching from vial-and-syringe delivery to disposable pens. ${ }^{11,12}$ Those that have studied the effect of switching analyzed treatment adherence, resource utilization, and associated healthcare costs but did not include glycosylated hemoglobin (A1C) as an end point. Furthermore, they used a time frame of July 2001-December 2002, which does not capture the newer insulin pens, such as insulin glargine SoloSTAR ${ }^{\circledR}$ (sanofi-aventis U.S., Bridgewater, NJ). Therefore more up-to-date studies are needed that include A1C as a parameter of glycemic control and incorporate newer insulin pen devices.

Evidence derived from studies in which patients initiate therapy with an insulin glargine pen compared with insulin

${ }^{1}$ STATinMED Research, Inc., Ann Arbor, Michigan.

${ }^{2}$ sanofi-aventis U.S., Bridgewater, New Jersey.

${ }^{3}$ Pro Unlimited, Boca Raton, Florida.

${ }^{4}$ The University of Michigan, Ann Arbor, Michigan. 
glargine vial-and-syringe cannot be readily applied to patients who switch from insulin glargine vial-and-syringe to insulin glargine pens because the switchers are likely to have different baseline characteristics and face different challenges compared with initiators. Therefore, a study on clinical and economic outcomes of patients switching from insulin glargine vial-and-syringe to insulin glargine pens is required.

The current study aims to evaluate the real-world clinical and economic outcomes among patients who were already administering insulin glargine therapy via vial-and-syringe who then switched to a prefilled disposable insulin glargine pen device (SoloSTAR).

\section{Subjects and Methods}

This retrospective analysis evaluated data from the national managed-care IMPACT ${ }^{\circledR}$ database (Ingenix Inc., Salt Lake City, UT). This database includes medical and pharmacy claims, demographic data, and limited laboratory results for more than 86 million members from over 40 healthcare plans. Clinical and economic outcomes were compared between individuals who had converted from administering glargine via vial-and-syringe to the pen device (Switchers) and patients who continued to use vial-and-syringe administration (Continuers).

\section{Study design}

Retrospective data were evaluated for adults diagnosed with T2DM included in the IMPACT database during prespecified dates (between July 2006 and December 2010). Eligible participants were to have had at least one inpatient or two outpatient physician visits dated $\geq 30$ days apart with a primary or secondary diagnosis of International Classification of Diseases, $9^{\text {th }}$ Revision, Clinical Modification (ICD9-CM) code $250 . x 0$ or $250 . x 2$. In addition, participants were required to have received at least two prescriptions for an insulin glargine vial (GLA-V) between July 2006 and December 2010.

Eligible participants were divided into Switchers and Continuers, based on insulin glargine use. Switchers were identified as patients who filled at least one prescription for an insulin glargine prefilled disposable pen (GLA-P), whereas the Continuers never filled a prescription for GLA-P during the study period. For the Switcher cohort, the index date was defined as the date of the first prescription for GLA-P. To allow a fair comparison on persistency and outcomes between the Switcher and Continuer groups, a GLA-V claim was selected randomly as the index date for the Continuer group, starting from the third GLA-V claim during the study period. For both cohorts, eligible participants were required to have continuous healthcare plan enrollment with medical and pharmacy benefits for at least 6 months before the index date (defined as the baseline period) and 1 year after the index date (defined as the follow-up period). Participants were also required to have had at least one GLA-V claim in each quarter and at least one $\mathrm{A} 1 \mathrm{C}$ test result during the baseline period.

\section{Analyses and study end points}

Various data were retrieved from the database, including patient demographics, A1C values, comorbidities, and medications. Each patient was assigned a Charlson Comorbidity
Index score, calculated by assigning disease severity weights of $1-6$ to 19 potential comorbid conditions. ${ }^{16}$ Baseline rapidacting insulin use was used to assign participants to a category of no rapid-acting insulin, rapid-acting insulin vial, or rapid-acting insulin pen. Baseline data were also collected for hypoglycemia occurrence, healthcare costs and utilization, and co-payments.

Outcome measures included treatment persistence and adherence and clinical and economic outcomes. Treatment persistence with insulin therapy is difficult to measure because of its nonfixed dosing. In previously published studies, an empirical approach was used to estimate insulin treatment persistence using two measures: (1) 1-year followup treatment persistence as dichotomous measures and (2) treatment persistence days within 1-year follow-up as continuous measures. ${ }^{15,17-19}$ During the 1-year follow-up from index date, patients were considered persistent with the treatment if they remained on glargine therapy without discontinuation or switching. Study medication was considered to have been discontinued if the prescription was not refilled within the expected time of medication coverage, defined as the $90^{\text {th }}$ percentile of the time, stratified by the metric quantity supplied between first and second fills among patients with at least one refill. ${ }^{15}$ The parameter treatment persistence days was defined as number of days from the index date until the treatment discontinuation/ switching date. Sensitivity analyses were also conducted using the $75^{\text {th }}$ and $95^{\text {th }}$ percentiles of the time. Adherence to insulin therapy was assessed as the adjusted medication possession ratio (MPR), which takes into account the differences in insulin device package size, during the 1-year follow-up. ${ }^{20}$ Insulin package sizes may vary in volume (10 $\mathrm{mL}$ for vial, $5 \times 3 \mathrm{~mL}$ for pen, etc.). As the total volume per package for pens is typically $50 \%$ larger than for vials, this will result in longer average times between refills for pen users (and vice versa), while the days' supply information recorded in the pharmacy claims may be similar across pen and vial users. To correct for the difference in refill times, the MPR was adjusted as follows: with the traditional MPR defined as the total day's supply of all filled study drug prescriptions in the analysis period divided by the number of days in the analysis period, the adjusted MPR for the pen group was calculated as the (traditional $\mathrm{MPR}) \times($ average days between prescription refills for patients using a pen/average days of supply for patients using a pen). For the vial-and-syringe group, (traditional $\mathrm{MPR}) \times($ average days between prescription refills for patients using vials/average days of supply for patients using vial-and-syringe) was used.

Clinical outcomes included 1-year change in A1C from baseline, hypoglycemia, and daily average consumption (DACON) of insulin glargine. Hypoglycemia was defined as a healthcare encounter (outpatient, inpatient, or emergency room [ER] visit) with a primary or secondary ICD-9-CM diagnosis code for hypoglycemia (ICD-9-CM code 250.8 for diabetes with other specified manifestations, 251.0 for hypoglycemic coma, 251.1 for other specified hypoglycemia, or 251.2 for hypoglycemia, unspecified). ${ }^{21}$ The setting of the hypoglycemic event (outpatient, ER, or hospital) was used as proxy for severity of the event. Hypoglycemia was assessed by both the prevalence rate of hypoglycemia-related events (defined as the percentage of patients with at least one 
hypoglycemia-related event occurring during the 1-year follow-up period) and the rate of hypoglycemic events (defined as the number of new cases of hypoglycemia-related events per 100 patients per year). DACON was calculated as the total number of units dispensed before the last refill divided by the total number of days between initiation and the last refill during the 1-year follow-up period.

Economic outcomes included healthcare utilization and cost. Healthcare utilizations were based on the number of hospitalizations, ER and office visits, and inpatient days. Allcause and diabetes-related health care costs in the follow-up period were calculated for inpatient, outpatient, ER, prescription, and total costs. Diabetes supply costs were also calculated. Diabetes-related healthcare resource utilization included claims with a primary or secondary diagnosis of diabetes (ICD-9-CM of 250.xx). Diabetes-related healthcare costs included costs from medical claims with a primary or secondary diagnosis of diabetes (ICD-9-CM of 250.xx), antidiabetes medications, glucose meters, and test strips.

\section{Statistical analyses}

Before we undertook multivariate analyses comparing the outcomes of treatment in the Switcher and Continuer cohorts, patients from each cohort were matched using propensity score matching at a 1:1 ratio to control for confounders. ${ }^{22}$ A patient from one cohort can only be matched to a patient from the other cohort if their propensity scores-fitted values of the probability of being a member of the overall cohort-are within \pm 0.01 units of one another. Patients who cannot be matched will be dropped from the analysis. Patients in the Switcher and Continuer cohorts were matched by the following baseline characteristics: age, baseline A1C, co-payment, initial year, healthcare plan type, geographic region, participation in diabetes education programs, baseline DACON, baseline and adjusted MPR, Charlson Comorbidity Index score, number of endocrinologist visits, any endocrinologist visits, number of oral antidiabetes drug types (metformin, thiazolidinediones, sulfonylureas, $\alpha$-glucosidase inhibitors, and dipeptidyl peptidase- 4 inhibitors), diuretics, use of sulfonylureas, any office visit, hypertension, and diabetes supply cost. Among matched patients, baseline characteristics and clinical and economic outcomes were summarized and compared, with $P$ values provided by Student's $t$ tests for continuous variables or $\chi^{2}$ tests for categorical variables. Time to discontinuation was analyzed using Kaplan-Meier analysis. Cox regression was used to estimate the impact of switching to pen versus continuing vial-and-syringe delivery on time to treatment discontinuation of glargine.

\section{Results}

In total, 3,893 eligible patient records were identified from the database: 665 patients were included in the Switcher cohort, and 3,228 patients were included in the Continuer cohort. After propensity score matching, 603 patients in the Switcher cohort were matched with an equivalent number of patients in the Continuer cohort. The remaining 52 patients in the Switcher cohort could not be so matched and were excluded from the analysis, as were the remaining unmatched patients in the Continuer cohort. Baseline characteristics of the matched cohorts are shown in Table 1.
Table 1. Baseline Patient Characteristics of the Matched Switcher and Continuer Cohorts

\begin{tabular}{|c|c|c|c|}
\hline Characteristic & $\begin{array}{l}\text { Pen switchers } \\
\quad(\mathrm{n}=603)\end{array}$ & $\begin{array}{l}\text { Vial continuers } \\
\quad(\mathrm{n}=603)\end{array}$ & $\begin{array}{c}\mathrm{P} \\
\text { value }^{\mathrm{a}}\end{array}$ \\
\hline Mean (SD) age (years) & $52.56(11.53)$ & $52.95(11.52)$ & 0.5587 \\
\hline $\operatorname{Men}[n(\%)]$ & $336(55.7)$ & $352(58.4)$ & 0.3520 \\
\hline A1C (\%) [mean (SD)] & $8.46(1.85)$ & $8.39(1.73)$ & 0.5141 \\
\hline CCI score [mean (SD)] & $0.75(1.47)$ & $0.76(1.48)$ & 0.9378 \\
\hline \multicolumn{4}{|l|}{ Comorbidities $[n(\%)]$} \\
\hline Hyperlipidemia & $421(69.8)$ & $432(71.6)$ & 0.4863 \\
\hline Hypertension & $387(64.2)$ & $388(64.3)$ & 0.9521 \\
\hline Retinopathy & $101(16.8)$ & $107(17.7)$ & 0.6474 \\
\hline Neuropathy & $99(16.4)$ & $83(13.8)$ & 0.1981 \\
\hline Mental illness & $84(13.9)$ & $88(14.6)$ & 0.7419 \\
\hline $\begin{array}{l}\text { Chronic pulmonary } \\
\text { disease }\end{array}$ & $55(9.1)$ & $58(9.6)$ & 0.7669 \\
\hline Renal disease & $57(9.5)$ & $45(7.5)$ & 0.2143 \\
\hline Nephropathy & $43(7.1)$ & $55(9.1)$ & 0.2060 \\
\hline $\begin{array}{l}\text { Congestive heart } \\
\text { failure }\end{array}$ & $46(7.6)$ & 39 & 0.4310 \\
\hline $\begin{array}{l}\text { Peripheral vascular } \\
\text { disease }\end{array}$ & $45(7.5)$ & 34( & 0.2005 \\
\hline \multicolumn{4}{|c|}{ Oral antidiabetic drugs $[n(\%)]$} \\
\hline Metformin & $240(39.8)$ & $(40.0)$ & 0.9531 \\
\hline Sulfonylureas & $132(21.9)$ & 130 & 0.8889 \\
\hline Thiazolidinediones & $112(18.6)$ & $114(18.9)$ & 0.8827 \\
\hline $\begin{array}{l}\text { Dipeptidyl } \\
\text { peptidase-4 } \\
\text { inhibitors }\end{array}$ & $49(8.1)$ & $46(7.6)$ & 0.7485 \\
\hline $\begin{array}{l}\text { Glucagon-like } \\
\text { peptide-1 agonists }\end{array}$ & $45(7.5)$ & $38(6.3)$ & 0.4259 \\
\hline Meglitinides & $14(2.3)$ & $19(3.2)$ & 0.3775 \\
\hline \multicolumn{4}{|l|}{ RAI indicator $[n(\%)]$} \\
\hline No RAI & $264(43.8)$ & $261(43.3)$ & 0.8617 \\
\hline RAI vial & $111(18.4)$ & $116(19.2)$ & 0.7126 \\
\hline RAI pen & $228(37.8)$ & $226(37.5)$ & 0.9054 \\
\hline $\begin{array}{l}\text { Insulin DACON (units) } \\
\text { [mean (SD)] }\end{array}$ & $40.60(23.64)$ & $41.32(23.18)$ & 0.5928 \\
\hline Mean (SD) MPR & 0.71 & 0.71 & 0.9029 \\
\hline $\begin{array}{l}\text { Mean (SD) adjusted } \\
\text { MPR }\end{array}$ & $0.86(0.18)$ & $0.86(0.17)$ & 0.9234 \\
\hline \multicolumn{4}{|c|}{ Hypoglycemia rates $[n(\%)]$} \\
\hline Any hypoglycemia & $44(7.3)$ & $42(7.0)$ & 0.8229 \\
\hline $\begin{array}{c}\text { Any inpatient/ER } \\
\text { hypoglycaemia }\end{array}$ & $24(4.0)$ & $15(2.5)$ & 0.1429 \\
\hline \multicolumn{4}{|l|}{ Mean $(\mathrm{SD})$ costs $(\$)$} \\
\hline All-cause cost (total) & $9,849(17,969)$ & $9,589(14$, & 0.785 \\
\hline Inpatie & $2,764(13,011)$ & $2,527(10,854)$ & 0.7313 \\
\hline Outpatient cost & $3,635(7,326)$ & $3,606(6,849)$ & 0.9419 \\
\hline ER cost & $352(1,222)$ & $270(911)$ & 0.188 \\
\hline Prescription cost & $3,098(2,593)$ & $3,187(3,113)$ & 0.5885 \\
\hline $\begin{array}{l}\text { Diabetes-related } \\
\text { cost (total) }\end{array}$ & $4,071(7,191)$ & $3,921(5,966)$ & 0.6945 \\
\hline Inpatient cost & $1,155(5,739)$ & $1,164(5,219)$ & 0.9778 \\
\hline Outpatient cost & $1,213(3,354)$ & $1,039(2,065)$ & 0.2780 \\
\hline ER cost & $157(726)$ & $144(612)$ & 0.7277 \\
\hline Prescriptic & 1,290 (909) & 1,318 (950) & 0.5965 \\
\hline $\begin{array}{l}\text { Diabetes supply } \\
\text { cost }\end{array}$ & $256(292)$ & $257(294)$ & 0.9549 \\
\hline
\end{tabular}

The baseline period was defined as 6 months prior to the index date.

${ }^{a} P$ values provided by Student's $t$ tests for continuous variables or $\chi^{2}$ tests for categorical variables.

A1C, glycosylated hemoglobin A1C; CCI, Charlson Comorbidity Index; DACON, daily average consumption; ER, emergency room; MPR, medication possession ratio; RAI, rapid-acting insulin. 
All baseline characteristics were comparable for the two cohorts, with the matched sample used for all subsequent analyses.

\section{Persistence and adherence}

During the 1-year follow-up period, patients in the Switcher cohort had significantly higher persistence than patients in the Continuer cohort $(65.3 \%$ vs. $49.8 \% ; P<0.0001)$. This finding was confirmed by sensitivity analyses conducted at the $75^{\text {th }}(39.6 \%$ vs. $26.4 \%, P<0.0001)$ and $95^{\text {th }}(78.4 \%$ vs. $61.4 \%, P<0.0001)$ percentiles. The number of treatment persistence days was also significantly higher for patients in the Switcher cohort (299.9 \pm 94.9 days) compared with the Continuer cohort $(264.0 \pm 113.6$ days $)(P<0.0001)$. Based on adjusted MPR, patients in the Switcher cohort were also significantly more adherent than those in the Continuer cohort ( 0.79 vs. $0.76 ; P=0.0173)$.

Kaplan-Meier analysis showed that Continuers were more likely to discontinue earlier than Switchers during the 1-year follow-up (log-rank test $P<0.0001$; Fig. 1 ). These results were confirmed by Cox regression analysis; after adjusting for other factors, Switchers were less likely to discontinue early than Continuers (hazard ratio of $0.544 ; P<0.0001$ ).

\section{Clinical outcomes}

DACON of insulin did not differ for patients in the Switcher (42.8 units) and Continuer (42.3 units) cohorts $(P=0.7757)$. A1C values were available for 350 matched Switcher and Continuer patients. The respective change in A1C from baseline was similar for Switchers and Continuers $(-0.04 \%$ and $-0.24 \%$, respectively; $P=0.0899)$. A similar percentage of patients in the Switcher and Continuer cohorts had a followup A1C value of $<7.0 \%(24.9 \%$ vs. $22.3 \%$; $P=0.4229)$.

Hypoglycemia incidence rates were also similar for the two cohorts: any hypoglycemic event occurred in $10.8 \%$ of
Switchers and $11.6 \%$ of Continuers $(P=0.6479)$, with a hypoglycemia-related hospitalization or ER visit reported for $3.8 \%$ of Switchers and $4.6 \%$ of Continuers $(P=0.4743)$. The average number of hypoglycemic events per 100 patient-years was 43 in Switchers and 31 in Continuers $(P=0.3648)$, and the average number of hypoglycemia-related hospitalizations or ER visits per 100 patient-years was six for both Switchers and Continuers $(P=0.8702)$.

\section{Healthcare utilization and costs}

Overall and diabetes-related healthcare utilizations during the 1-year follow-up period are summarized in Table 2 . There were no statistical differences in healthcare utilization between the two cohorts.

The overall and diabetes-related healthcare costs incurred during the 1-year follow-up period are shown in Table 2. Switchers and Continuers had similar overall $(\$ 20,870 \pm 30,534$ vs. $\$ 20,747 \pm 29,803 ; P=0.9434)$ and diabetesrelated $(\$ 8,333 \pm 10,344$ vs. $\$ 8,494 \pm 11,284 ; P=0.7972)$ total costs. As expected, diabetes-related prescription costs were significantly higher for Switchers compared with Continuers $(\$ 3,393 \pm 2,351$ vs. $\$ 2,889 \pm 2,035 ; P=0.0001)$. However, this higher prescription cost was offset by lower overall and diabetes-related outpatient and inpatient costs among Switchers than Continuers.

\section{Discussion}

This retrospective study of the IMPACT database showed significant improvements over a 1-year follow-up period in treatment persistence and adherence among patients who were treated with insulin glargine for T2DM and who switched from vial-and-syringe to prefilled disposable pen administration compared with patients who continued to use vial-and-syringe delivery. Clinical outcomes were similar between the two groups, which is similar to a previously

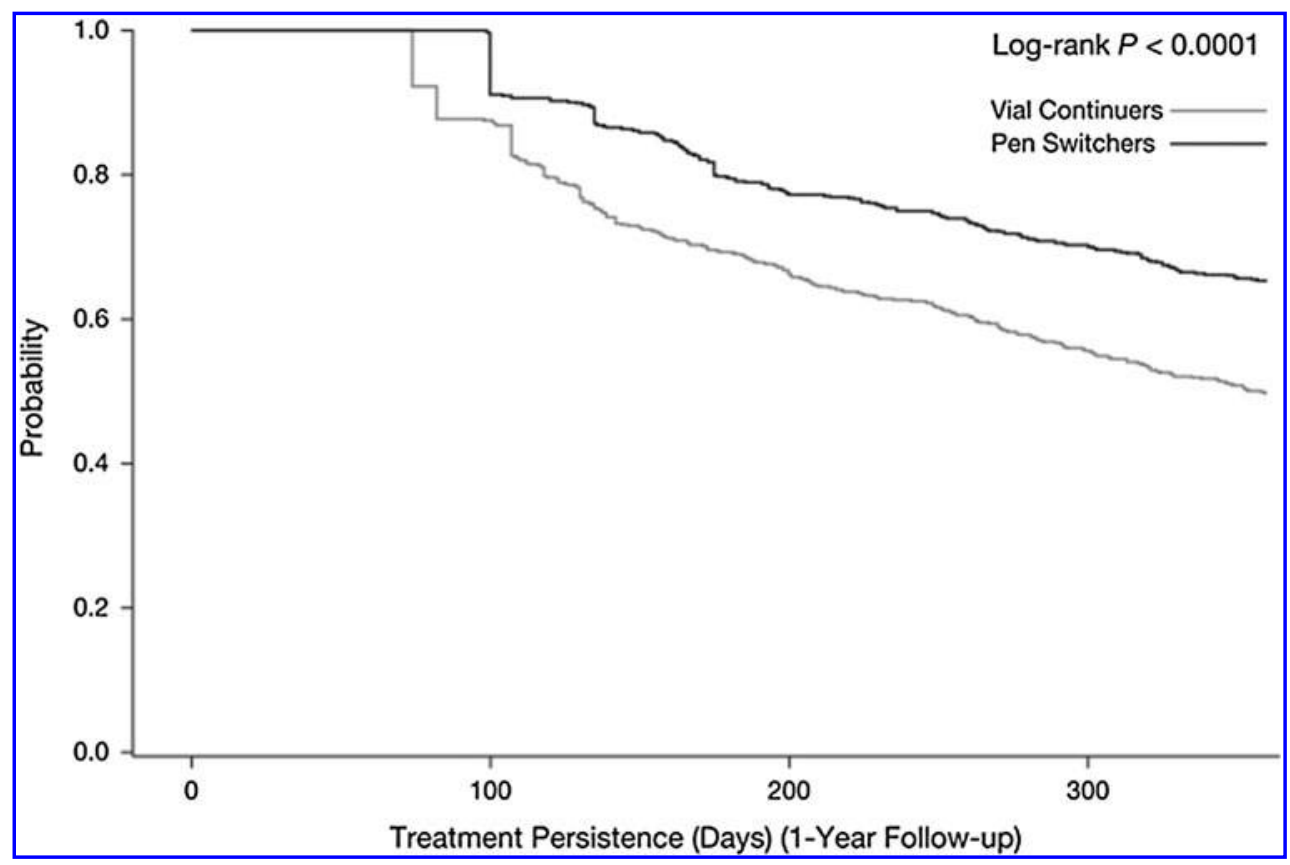

FIG. 1. Kaplan-Meier curve of time to discontinuation of all glargine use for the matched Switcher and Continuer cohorts. 
Table 2. Healthcare Costs and Utilizations of the Matched Switcher and Continuer Cohorts at the End of the 1-Year Follow-Up Period

\begin{tabular}{|c|c|c|c|}
\hline Utilization & Pen switchers $(\mathrm{n}=603)$ & Vial continuers $(\mathrm{n}=603)$ & $\mathrm{P}$ value ${ }^{\mathrm{a}}$ \\
\hline \multicolumn{4}{|l|}{ Overall healthcare utilization } \\
\hline Any hospitalization $[n(\%)]$ & $103(17.1)$ & $111(18.4)$ & 0.5465 \\
\hline Hospitalizations per patient $[n(\mathrm{SD})]$ & $0.27(0.69)$ & $0.29(0.76)$ & 0.6346 \\
\hline Hospitalization days [ $n$ (SD)] & $2.05(8.26)$ & $2.09(8.80)$ & 0.9301 \\
\hline Any ER visit $[n(\%)]$ & $215(35.7)$ & $187(31.0)$ & 0.0872 \\
\hline ER visits per patient $[n(S D)]$ & $0.85(2.33)$ & $0.87(2.54)$ & 0.8966 \\
\hline Any office visit $[n(\%)]$ & $601(99.7)$ & $601(99.7)$ & 1.0000 \\
\hline Office visits per patient $[n$ (SD)] & $18.34(17.36)$ & $18.41(17.18)$ & 0.9376 \\
\hline Any endocrinologist visit $[n(\%)]$ & $288(47.8)$ & $277(45.9)$ & 0.5256 \\
\hline Endocrinologist visits per patient $[n(\mathrm{SD})]$ & $1.59(2.21)$ & $1.59(2.22)$ & 0.9896 \\
\hline \multicolumn{4}{|l|}{ Diabetes-related healthcare utilization } \\
\hline Any hospitalization [n (\%)] & $93(15.4)$ & $100(16.6)$ & 0.5825 \\
\hline Hospitalizations per patient $[n(\mathrm{SD})]$ & $0.22(0.61)$ & $0.22(0.57)$ & 0.9608 \\
\hline Hospitalization days [ $n$ (SD)] & $1.69(7.47)$ & $1.73(8.25)$ & 0.9185 \\
\hline Any ER visit $[n(\%)]$ & $149(24.7)$ & $141(23.4)$ & 0.5899 \\
\hline ER visits per patient $[n(\mathrm{SD})]$ & $0.38(0.86)$ & $0.41(1.07)$ & 0.6158 \\
\hline Any office visit $[n(\%)]$ & $597(99.0)$ & $591(98.0)$ & 0.1542 \\
\hline Office visits per patient $[n(\mathrm{SD})]$ & $8.01(7.63)$ & $7.91(6.58)$ & 0.8116 \\
\hline \multicolumn{4}{|l|}{ Mean (SD) overall healthcare costs (\$) } \\
\hline Total cost & $20,870(30,534)$ & $20,747(29,803)$ & 0.9434 \\
\hline Inpatient cost & $5,276(18,096)$ & $5,515(18,080)$ & 0.8182 \\
\hline Outpatient cost & $7,699(16,958)$ & $7,921(16,114)$ & 0.8160 \\
\hline ER cost & $652(2,359)$ & $646(1,867)$ & 0.9646 \\
\hline Prescription cost & $7,244(5,674)$ & $6,665(6,038)$ & 0.0863 \\
\hline \multicolumn{4}{|l|}{ Mean (SD) diabetes-related healthcare costs (\$) } \\
\hline Total cost & $8,333(10,344)$ & $8,494(11,284)$ & 0.7972 \\
\hline Inpatient cost & $2,048(8,213)$ & $2,366(7,670)$ & 0.4876 \\
\hline Outpatient cost & $2,068(3,440)$ & $2,380(6,835)$ & 0.3165 \\
\hline ER cost & $271(1,087)$ & $311(1,201)$ & 0.5511 \\
\hline Prescription cost & $3,393(2,351)$ & $2,889(2,035)$ & 0.0001 \\
\hline Diabetes supply cost & $553(632)$ & $548(592)$ & 0.8943 \\
\hline
\end{tabular}

${ }^{\text {a }} P$ values provided by Student's $t$ tests for continuous variables or $\chi^{2}$ tests for categorical variables.

ER, emergency room.

reported randomized comparative trial with pen versus vialand-syringe for insulin aspart administration. ${ }^{8}$ Furthermore, improved treatment persistence and adherence were achieved without a significant increase in healthcare utilization or total costs. For healthcare payers, such as health insurance companies, these findings imply that disposable pen devices for insulin administration may be a more costeffective option than the traditional vial-and-syringe, in that the marginal benefits of these devices outweigh their marginal costs.

In an observational study conducted in patients with type 1 or 2 diabetes who had recently switched to or started insulin treatment using insulin glargine by pen, at least $95 \%$ of patients rated pen use as either good or excellent for ease of use, learning to use, and selecting and reading the dose. The study data suggest that, despite major changes in therapy, the new treatment and administration methods were well accepted by patients. ${ }^{23}$ Intuitively, it would follow that changes in patient perception in these areas may lead to improvements in persistence and adherence. Data from the current study support previously published studies showing improved adherence and persistence when initiating insulin treatment using a pen delivery system rather than vial-and-syringe ${ }^{15,17,24}$ or after switching from vial-and-syringe administration to pen delivery. ${ }^{11,12}$ Although some studies have reported a reduc- tion in the risk of hypoglycemia following switching to pen delivery, ${ }^{1,5,11,12}$ glycemic control was similar between patients using pen and vial-and-syringe delivery systems in the current study.

Previously published data have shown that the use of pens may contribute to improved glycemic control. ${ }^{15}$ The use of pen devices may indirectly contribute to improved $\mathrm{A} 1 \mathrm{C}$ levels by increasing patient adherence and satisfaction ${ }^{14}$ or by improving accuracy of administration. ${ }^{15,25}$ Improved glycemic control, however, was not observed after switching to pen administration in the current study. Furthermore, in contrast to other studies, ${ }^{11,12}$ the current study did not observe any reduction in the incidence of hypoglycemia and associated healthcare utilizations among patients who switched to a pen device compared with those continuing with a vial-and-syringe method. Cobden et al. ${ }^{12}$ reported a significantly decreased risk of hypoglycemia-attributable ER visits (odds ratio [OR] 0.36, 95\% confidence interval [CI] $0.16-0.84$ ) and physician visits (OR $0.39,95 \%$ CI $0.20-0.77$ ) if patients switched from vial-and-syringe to pen device usage for the delivery of insulin. Lee et al. ${ }^{11}$ likewise reported that the likelihood of experiencing a hypoglycemic event was significantly reduced after patients had switched to using a pen device for the delivery of insulin (OR 0.50,95\% CI $0.37-0.67)$. This discrepancy may be due to the different 
study designs. Cobden et al. ${ }^{12}$ and Lee et al. ${ }^{11}$ used a simple pre-post comparison approach in their articles, whereas the current study used a more robust controlled cohort analysis with propensity score matching. Instead of comparing with the pre-switching period, the current study used a method in which the hypoglycemia rate for Switchers during the follow-up period was compared with that in the Continuer group. Both groups had low rates of hypoglycemia; therefore, the sample size may have been too small to detect significant differences.

This study has several limitations. The IMPACT database comprises claims submitted by healthcare providers to insurance companies for reimbursement on behalf of the employees of client companies and may be subject to coding errors and missing data. The population sample considered in this retrospective analysis is based on a large convenience sample, which by its nature is nonrandom and therefore may be subject to bias, limiting the generalizability of the findings to similar populations and treatments. ${ }^{24}$ Also, the use of pharmacy claims data and patient medical histories to measure adherence and persistence may not provide sufficiently detailed information to ensure that prescribed medications are being appropriately used. In addition, as in any retrospective study, causality between treatment and differences in outcomes cannot be established. Furthermore, our study is limited to only 1 year of follow-up, and significant differences may have been noted if longer follow-up had been used. Considering that $\mathrm{A} 1 \mathrm{C}$ is an important health outcome for T2DM, another limitation of this analysis is the fact that A1C data were only available for a proportion of patients (350 of 603). Finally, the use of pen devices has been shown to facilitate insulin administration in patients with visual impairments or restricted manual dexterity-for example, elderly patients. ${ }^{5,26,27}$ Our study did not compare outcomes among patients based on age or visual impairments, although this might be considered as a potentially important contributing factor in subsequent studies.

In conclusion, this real-world study has shown that switching from vial-and-syringe to a pen device for insulin glargine administration resulted in increased treatment persistence and adherence with similar clinical outcomes. Despite the higher diabetes-related prescription costs associated with the switch to pen use, there was no increase in total healthcare costs, suggesting that the switch to pen use is a cost-effective option for improving insulin persistence and adherence. These findings may assist with optimizing the management of patients with T2DM.

\section{Acknowledgments}

This study was funded by sanofi-aventis U.S., Inc. The authors received editorial/writing support in the preparation of this manuscript from Tessa Hartog, PhD, of Excerpta Medica, funded by sanofi-aventis U.S., Inc.

\section{Author Disclosure Statement}

L.X. and O.B. are employees at STATinMED Research, which received funding to carry out this work from sanofiaventis U.S., Inc. S.Z., W.W., and J.G. are employees at sanofiaventis U.S., Inc. C.P. is an employee at PRO Unlimited, which received funding to carry out this work from sanofi-aventis U.S., Inc.

\section{References}

1. Brunton S: Initiating insulin therapy in type 2 diabetes: benefits of insulin analogs and insulin pens. Diabetes Technol Ther 2008;10:247-256.

2. DeWitt DE, Hirsch IB: Outpatient insulin therapy in type 1 and type 2 diabetes mellitus: scientific review. JAMA 2003;289:2254-2264.

3. Inzucchi SE, Bergenstal RM, Buse JB, Diamant M, Ferrannini E, Nauck M, Peters AL, Tsapas A, Wender R, Matthews DR: Management of hyperglycaemia in type 2 diabetes: a patient-centered approach. Position statement of the American Diabetes Association (ADA) and the European Association for the Study of Diabetes (EASD). Diabetologia 2012;55: 1557-1596.

4. Korytkowski M, Niskanen L, Asakura T: FlexPen: addressing issues of confidence and convenience in insulin delivery. Clin Ther 2005;27(Suppl B):S89-S100.

5. Goldstein HH: Pen devices to improve patient adherence with insulin therapy in type 2 diabetes. Postgrad Med 2008;120:172-179.

6. Magwire ML: Addressing barriers to insulin therapy: the role of insulin pens. Am J Ther 2011;18:392-402.

7. Peyrot M, Rubin RR, Lauritzen T, Skovlund SE, Snoek FJ, Matthews DR, Landgraf R, Kleinebreil L; International DAWN Advisory Panel: Resistance to insulin therapy among patients and providers: results of the cross-national Diabetes Attitudes, Wishes, and Needs (DAWN) study. Diabetes Care 2005;28:2673-2679.

8. Korytkowski M, Bell D, Jacobsen C, Suwannasari R: A multicenter, randomized, open-label, comparative, twoperiod crossover trial of preference, efficacy, and safety profiles of a prefilled, disposable pen and conventional vial/ syringe for insulin injection in patients with type 1 or 2 diabetes mellitus. Clin Ther 2003;25:2836-2848.

9. Carter J, Roberts A: Usability of a pre-filled insulin injection device in a 3-month observational survey of everyday clinical practice in Australia. Curr Med Res Opin 2008;24: 2741-2749.

10. Summers KH, Szeinbach SL, Lenox SM: Preference for insulin delivery systems among current insulin users and nonusers. Clin Ther 2004;26:1498-1505.

11. Lee WC, Balu S, Cobden D, Joshi AV, Pashos CL: Medication adherence and the associated health-economic impact among patients with type 2 diabetes mellitus converting to insulin pen therapy: an analysis of third-party managed care claims data. Clin Ther 2006;28:1712-1725.

12. Cobden D, Lee WC, Balu S, Joshi AV, Pashos CL: Health outcomes and economic impact of therapy conversion to a biphasic insulin analog pen among privately insured patients with type 2 diabetes mellitus. Pharmacotherapy 2007;27:948-962.

13. Pawaskar MD, Camacho FT, Anderson RT, Cobden D, Joshi $\mathrm{AV}$, Balkrishnan R: Health care costs and medication adherence associated with initiation of insulin pen therapy in Medicaid-enrolled patients with type 2 diabetes: a retrospective database analysis. Clin Ther 2007;29:1294-1305.

14. Asche CV, Shane-McWhorter L, Raparla S: Health economics and compliance of vials/syringes versus pen devices: a review of the evidence. Diabetes Technol Ther 2010;12(Suppl 1):S-101-S-108.

15. Davis SN, Wei W, Garg S: Clinical impact of initiating insulin glargine therapy with disposable pen versus vial in patients with type 2 diabetes mellitus in a managed care setting. Endocr Pract 2011;17:845-852. 
16. Charlson M, Szatrowski TP, Peterson J, Gold J: Validation of a combined comorbidity index. I Clin Epidemiol 1994;47: 1245-1251.

17. Xie L, Wei W, Pan C, Du J, Baser O: A real-world study of patients with type 2 diabetes initiating basal insulins via disposable pens. Adv Ther 2011;28:1000-1011.

18. Baser $\mathrm{O}$, Wei W, Baser E, Xie L: Clinical and economic outcomes in patients with type 2 diabetes initiating insulin glargine disposable pen versus exenatide BID. J Med Econ 2011;14:673-680.

19. Levin P, Wei W, Wang L, Pan C, Douglas D, Baser O: Combination therapy with insulin glargine and exenatide: real-world outcomes in patients with type 2 diabetes. Curr Med Res Opin 2012;28:439-446.

20. Baser O, Bouchard J, DeLuzio T, Henk H, Aagren M: Assessment of adherence and healthcare costs of insulin device (FlexPen) versus conventional vial/syringe. Adv Ther 2010;27:94-104.

21. Zhao Y, Campbell CR, Fonseca V, Shi L: Impact of hypoglycemia associated with antihyperglycemic medications on vascular risks in veterans with type 2 diabetes. Diabetes Care 2012;35:1126-1132.

22. Rosenbaum PR, Rubin DB: The central role of the propensity score in observational studies for causal effects. Biometrika 1983;70:41-55.
23. Hancu N, Czupryniak L, Genestin E, Sourij H: A Pan-European and Canadian prospective survey to evaluate patient satisfaction with the SoloSTAR insulin injection device in type 1 and type 2 diabetes. J Diabetes Sci Technol 2011;5:1224-1234.

24. Buysman E, Conner C, Aagren M, Bouchard J, Liu F: Adherence and persistence to a regimen of basal insulin in a pre-filled pen compared to vial/syringe in insulin-naïve patients with type 2 diabetes. Curr Med Res Opin 2011;27:1709-1717.

25. Clarke A, Dain MP: Dose accuracy of a reusable insulin pen using a cartridge system with an integrated plunger mechanism. Expert Opin Drug Deliv 2006;3:677-683.

26. Marcus A: Diabetes care-insulin delivery in a changing world. Medscape J Med 2008;10:120.

27. Shaghouly AA, Shah BR: The prescription of insulin pen devices versus syringes for older people with diabetes. Diabetes Technol Ther 2009;11:439-442.

Address correspondence to:

Steve Zhou, PhD

sanofi-aventis U.S.

55 Corporate Drive, Mailstop 55A-505B

Bridgewater, NJ 08807-5925

E-mail: Steve.Zhou@sanofi.com 


\section{This article has been cited by:}

1. Zhaoyang Li, Rachael Easton. 2017. Practical considerations in clinical strategy to support the development of injectable drugdevice combination products for biologics. mAbs 2008, 1-16. [Crossref]

2. Eric Zijlstra, Johannes Jahnke, Annelie Fischer, Christoph Kapitza, Thomas Forst. 2017. Impact of Injection Speed, Volume, and Site on Pain Sensation. Journal of Diabetes Science and Technology 10, 193229681773512. [Crossref]

3. Olga Horvat, Jelena Popržen, Ana Tomas, Milica Paut Kusturica, Zdenko Tomić, Ana Sabo. 2017. Factors associated with nonadherence among type 2 diabetic patients in primary care setting in eastern Bosnia and Herzegovina. Primary Care Diabetes . [Crossref]

4. Rosemarie Lajara, Eda Cengiz, Robert J. Tanenberg. 2017. The role of the new basal insulin analogs in addressing unmet clinical needs in people with type 1 and type 2 diabetes. Current Medical Research and Opinion 33:6, 1045-1055. [Crossref]

5. Wenhui Wei, Jenny Jiang, Youbei Lou, Sohini Ganguli, Mark S. Matusik. 2017. Benchmarking Insulin Treatment Persistence Among Patients with Type 2 Diabetes Across Different U.S. Payer Segments. Journal of Managed Care \& Specialty Pharmacy 23:3, 278-290. [Crossref]

6. Sergei Pechenov, Himanshu Bhattacharjee, Daniel Yin, Sachin Mittal, J. Anand Subramony. 2017. Improving drug-like properties of insulin and GLP-1 via molecule design and formulation and improving diabetes management with device \& drug delivery. Advanced Drug Delivery Reviews 112, 106-122. [Crossref]

7. Samuel Stolpe, Michel A. Kroes, Neil Webb, Tami Wisniewski. 2016. A Systematic Review of Insulin Adherence Measures in Patients with Diabetes. Journal of Managed Care \& Specialty Pharmacy 22:11, 1224-1246. [Crossref]

8. Pieralessandro Lasalvia, Julián Esteban Barahona-Correa, Diana Marcela Romero-Alvernia, Sebastián Gil-Tamayo, Camilo Castañeda-Cardona, Juan Gabriel Bayona, Juan José Triana, Andrés Felipe Laserna, Miguel Mejía-Torres, Paula RestrepoJimenez, Juliana Jimenez-Zapata, Diego Rosselli. 2016. Pen Devices for Insulin Self-Administration Compared With Needle and Vial. Journal of Diabetes Science and Technology 10:4, 959-966. [Crossref]

9. Philip Levin, Steve Zhou, Emily Durden, Amanda M. Farr, Jasvinder Gill, Wenhui Wei. 2016. Clinical and Economic Outcomes Associated With the Timing of Initiation of Basal Insulin in Patients With Type 2 Diabetes Mellitus Previously Treated With Oral Antidiabetes Drugs. Clinical Therapeutics 38:1, 110-121. [Crossref]

10. Grabner Michael, Peng Xiaomei, Caroline Geremakis, Jay Bae. 2015. Demographic and Clinical Profiles of Type 2 Diabetes Mellitus Patients Initiating Canagliflozin Versus DPP-4 Inhibitors in a Large U.S. Managed Care Population. Journal of Managed Care \& Specialty Pharmacy 21:12, 1204-1212. [Crossref]

11. Magaly Perez-Nieves, Dingfeng Jiang, Elizabeth Eby. 2015. Incidence, prevalence, and trend analysis of the use of insulin delivery systems in the United States (2005 to 2011). Current Medical Research and Opinion 31:5, 891-899. [Crossref]

12. Sheila Anne Doggrell, Vincent Chan. 2015. Adherence to insulin treatment in diabetes: can it be improved?\#\#\#\#\#\#\#\#\#\#\#\#\# \#\#\#\#\#\#\#\#\#\#. Journal of Diabetes 7:3, 315-321. [Crossref]

13. Xie Lin, Zhou Steve, Pinsky Brett W., Buysman Erin K., Baser Onur. 2014. Impact of Initiating Insulin Glargine Disposable Pen Versus Vial/Syringe on Real-World Glycemic Outcomes and Persistence Among Patients with Type 2 Diabetes Mellitus in a Large Managed Care Plan: A Claims Database Analysis. Diabetes Technology \& Therapeutics 16:9, 567-575. [Abstract] [Full Text HTML] [Full Text PDF] [Full Text PDF with Links] [Supplemental Material]

14. Hua Heng McVin Cheen, Seng Han Lim, Ming Chien Huang, Yong Mong Bee, Hwee Lin Wee. 2014. Adherence to Premixed Insulin in a Prefilled Pen Compared With a Vial/Syringe in People With Diabetes in Singapore. Clinical Therapeutics 36:7, 1043-1053. [Crossref]

15. Wenhui Wei, Steve Zhou, Raymond Miao, Chunshen Pan, Lin Xie, Onur Baser, Jasvinder Gill. 2014. Much Ado About Nothing? A Real-World Study of Patients with Type 2 Diabetes Switching Basal Insulin Analogs. Advances in Therapy 31:5, 539-560. [Crossref]

16. Wenhui Wei, Chunshen Pan, Lin Xie, Onur Baser. 2014. Real-World Insulin Treatment Persistence among Patients with Type 2 Diabetes. Endocrine Practice 20:1, 52-61. [Crossref]

17. Raymond Miao, Wenhui Wei, Jay Lin, Lin Xie, Onur Baser. 2014. Does Device Make Any Difference? A Real-world Retrospective Study of Insulin Treatment Among Elderly Patients With Type 2 Diabetes. Journal of Diabetes Science and Tecbnology 8:1, 150-158. [Crossref]

18. Sarah Thayer, Wenhui Wei, Erin Buysman, Lee Brekke, William Crown, Michael Grabner, Swetha Raparla, Ralph Quimbo, Mark J. Cziraky, Wenli Hu, Robert Cuddihy. 2013. The INITIATOR Study: Pilot Data on Real-World Clinical and Economic Outcomes in US Patients with Type 2 Diabetes Initiating Injectable Therapy. Advances in Therapy 30:12, 1128-1140. [Crossref] 
19. Keith L. Davis, Muralikrishna Tangirala, Juliana L. Meyers, Wenhui Wei. 2013. Real-world comparative outcomes of US type 2 diabetes patients initiating analog basal insulin therapy. Current Medical Research and Opinion 29:9, 1083-1091. [Crossref] 